\title{
Swarm Intelligence based Bee inspired routing protocol for multipath routing in MANET
}

\author{
M.Sumathi \\ Student, Network Engineering, Kalasalingam University, Virudhunagar, India
}

\begin{abstract}
MANET is one of the acts as routing. Routing main work is exchange information from one host to other host in the network. Multipath routing can be used to improve the efficiency of wireless links in Manet when sending multiple copies of data from one source to multiple group of receiver. In this paper BeeIP present multipath routing in Manet. BeeIP is swarm intelligence based new honeybee inspired routing protocol. It is based on the collective behavior of honeybee forager and able to actively detect multiple paths between Source and destination also distributes traffic across them. The simulation result show that proposed work achieves able to delivery of packet faster and average end to end delay and packet delivery ratio better than other routing protocol and this protocol maintains balanced control overhead during mobility changes.
\end{abstract}

Keywords: Swarm intelligence, Mobile ad hoc network, Ant-colony, Bee-colony, routing protocol.

\section{INTRODUCTION}

Mobile ad hoc networks (MANETs) are networks consist of all nodes are mobile and communicate with each other via wireless connections further nodes can join or leave at any time. It can change any location and configure itself and it is continuously self-configuring, infrastructure less and maintenance network of mobile devices connected without wires are built through the network. In a MANET, all the nodes are using wireless communication links to send and receive data packets also data must be routed via intermediate node and multi hop wireless links.

Further, it follows Packet Switched approach and evolving toward emulation of circuit switching. Finally due to the network limited resources, protocol need to provide energy consumption, and bandwidth and computing power.

\section{A) Challenges in Mobile Ad-hoc networks}

MANET has undergone many challenges at the time of routing. The main challenges are: 1. Limited power supply 2. Dynamically changing topology 3.Limited bandwidth 4.Security 5.Mobility-induced route changes 6.Mobility-induced packet losses 7.Battery constraints. So, it uses many applications such as laptop, cell phones, soldiers, meeting rooms, etc.

\section{B) Routing in Mobile Ad-Hoc Network}

Routing is the process of transfer instruction from one host to other host in a network. It is the mechanism of promote packets towards its Destination using most capable path. Energetic path is measured in various metrics like, Number of hops, traffic, security, etc. Main objective of routing is finding path with minimum overhead and also quick reconfiguration of broken path also to find and maintenance between nodes in a dynamic topology with desirable uni-directional links using minimum resources.

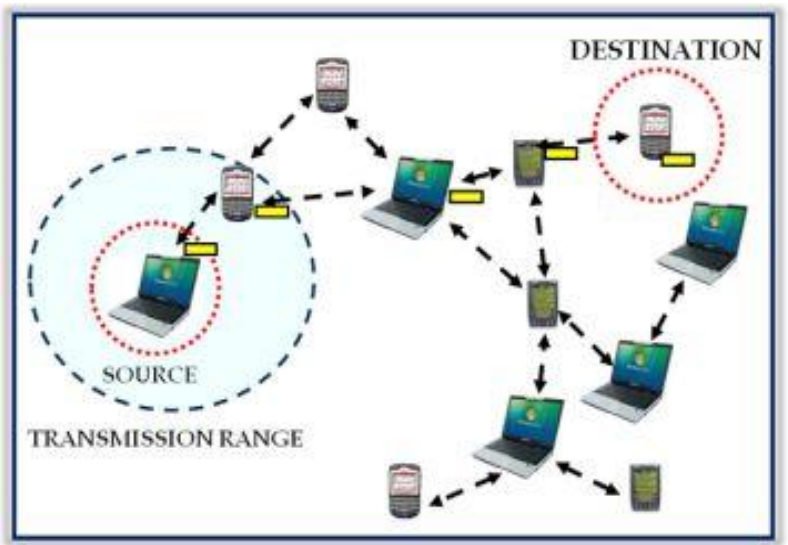

Figure.1. Mobile ad hoc Network

\section{C) Swarm Intelligence (SI)}

Bio-inspired routing protocol followed Swarm Intelligence approach, like Artificial Intelligence (AI). These approaches are given by utilizing agent based system inspired by the social behavior of natural systems, mostly found in insects. The agents allow the system to be self-organised, robust, efficient and scalable then achieve tasks that could not be done by a single agent at a global level. So It's follow some rules, although division of works and changing roles then routing protocol agents can dynamically change their behavior according to the different states of solving the routing problem.

Example in natural system of SI include ant colonies, honeybees, fish schooling. SI connection to designing network routing protocols are ant colony optimization (aco), bee colony optimization (bco).

1) Ant Colony Optimization (ACO): $\mathrm{ACO}$ is based on ant's behavior. ACO select the foraging behavior of real ants. It's follow unipath selection from nest to food. More specifically, this behavior in indirect communication between the ants by means of chemical pheromone trails, which setup them to find short paths between their nest and food sources. 


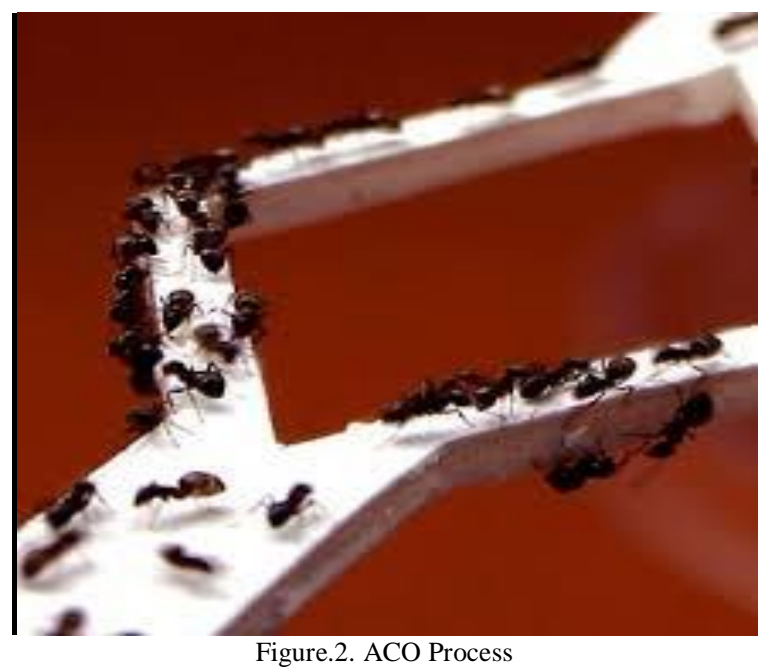

2) Bee Colony Optimization (BCO): Similar to ACO, The Bee Colony Optimization (BCO) is inspired by bees' behavior in the nature. The $\mathrm{BCO}$ scheme is creating multi agent system (colony of artificial bees) accomplished to solve difficult Optimization problems. The artificial bee colony performs partially like and partially differently from bee colonies in nature. Each artificial bee has to make a decision based on a certain probability. Finally, it uses direct communication to exchange information between bees.
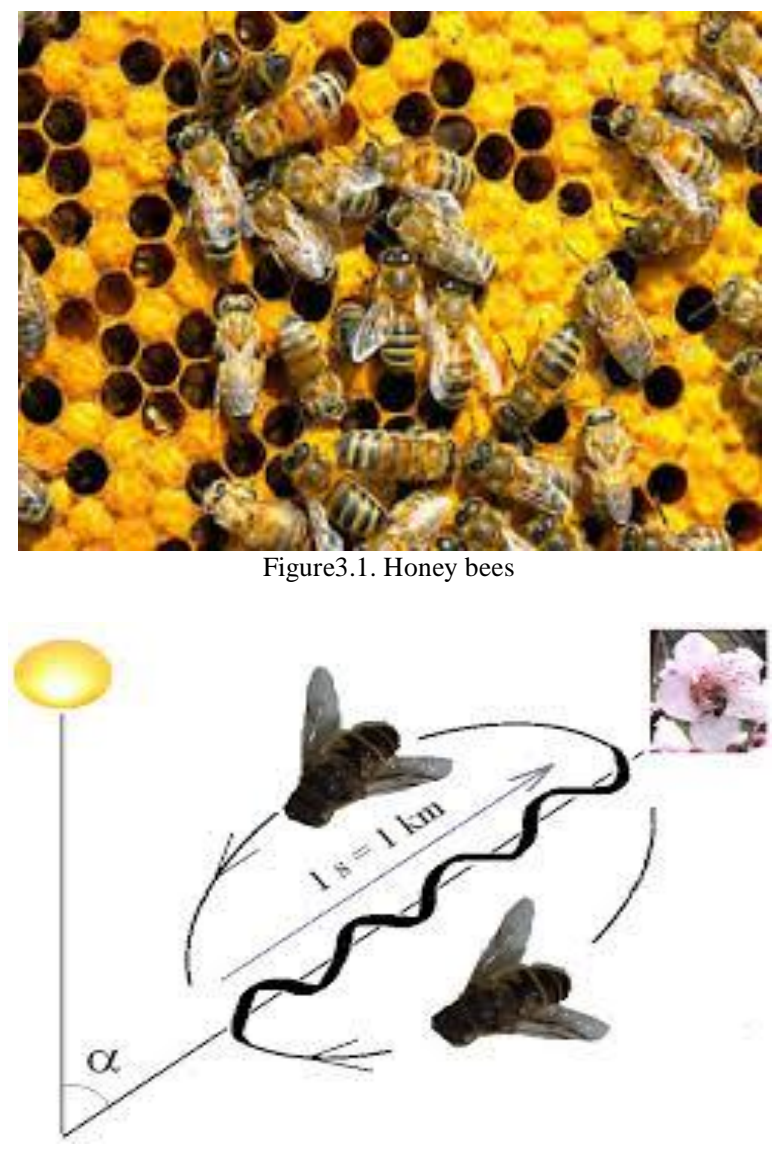

Figure3.2 BCO Process
In this paper present, proposed routing protocol for Bee-Inspired routing Protocol (BeeIP). It is based on swarm intelligence using multipath routing protocol in MANET. BeeIP follow Bee Colony Optimization technique (BCO) to solve difficult problem and improve the performance of AODV for the parameters like end-toend delay, throughput, packet delivery ratio, control overhead. So, BeeIP achieves faster data transmission and balanced control overhead compare than other routing protocol.

\section{RELATED WORK}

In this paper focus on AOMDV is an on-demand and multipath distance vector routing protocol for mobile ad hoc networks, it is based on the distance vector concept and uses hop-by-hop routing approach. Moreover, AOMDV finds routes on demand using a route discovery procedure. AOMDV protocol provides that multiple paths determine multiple loop-free, link disjoint path and efficiently finding paths using a flood-based route discovery. Simulation results have shown that AOMDV can outperform AODV, Performance of results are reduces the packet loss, end-to-end Delay and also reduces routing overhead than AODV.

In this paper author focus on AntHocNet is a hybrid multipath routing algorithm, designed on the principles of ACO routing. It's followed both reactive and proactive routing and does not maintain paths to all destinations at all times. First, it entire in a reactive path setup, where ant agents called reactive forward ants are sent by the source then to find multiple paths to the destination, and backward ant arrival to set up the paths. The paths are serving in pheromone tables indicating their respective quality. It's followed four mechanisms they are 1.Reactive path set up 2.Stochastic data routing 3.Proactive path probing, maintenance 4.link failure And achieved outperform AODV and simulation result has shown that high packet delivery ratio and average delay compare than AODV, AOMDV.

In this paper have presented BeeAdHoc is an energy efficient routing protocol. This algorithm is inspired by the foraging principle of honeybees it use less energy because it utilizes less control packets to do routing. This algorithm mainly utilizes two types of agents. They are Scouts, Forager, and Packer. It uses scout agents to discover routes between source and destination. When a route is required at the destination node, a scout agent is broadcast to the network. Once a scout entry to source node, the volunteer foragers creates and using bee dance does in nature. Certainly, a forager is the bee agent that receives data packets from a packer and delivers to the destination. BeeAdHoc consumes significantly less energy as compared to AODV, DSR and shown simulation result show that high packet delivery ratio, throughput and average delay compare than other protocol.

In this paper focus on BeeSensor is an even driven, reactive and on-demand multipath routing protocol. This 
algorithm is inspired by the foraging principle of honeybees. It works with four types of agents: Packers, Scouts, Forager, and Swarm. BeeSensor simulation results on deliver superior performance in terms of packet delivery ratio and latency, also this protocol use least $A$ energy compared with AODV and other SI algorithms.

In this paper have presented HOPNET, It is a hybrid in nature and consists of local proactive route discovery within neighbourhood and reactive communication between the neighbourhood. This network is divided into zone. The size of zone is not resolved locally but by the radius length measured in hops. Results indicate that HOPNET is highly scalable compared to AntHocNet. The results indicate that the selection of the zone has impact on packet delivery ratio and performs significantly better than AntHocNet for high and low mobility.

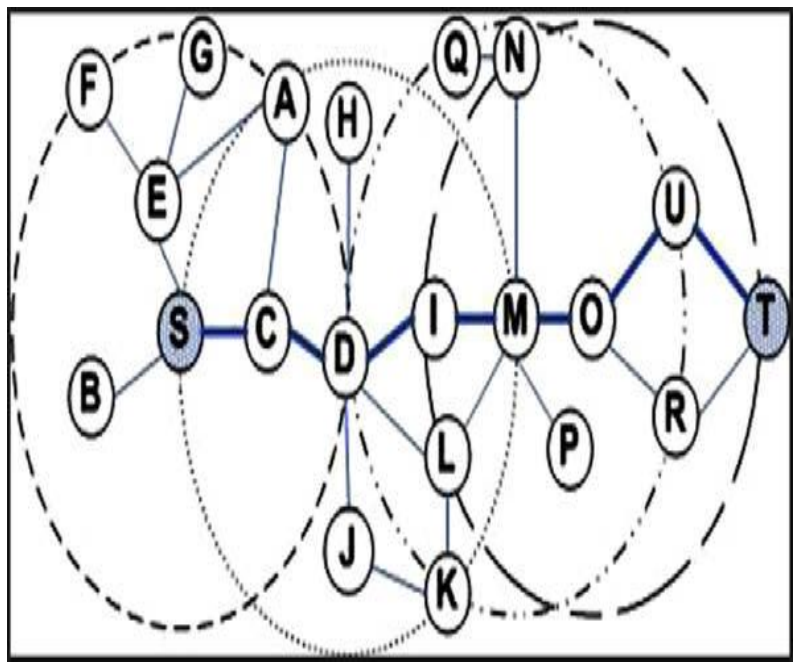

Figure.4. HOPNET Examples

\section{PROPOSED TECHNIQUE}

Bee-inspired protocol (BeeIP) is proposed protocol. BeeIP is a advanced honeybee-inspired adaptive routing protocol on the collective behaviour of honeybee forager, its follow reactive approach, agents analyse the topology only when data are required to be transmitted between nodes and agents are apt to adaptively work in parallel, transmit with each other to exchange simple information that is carefully collected from the network and achieve multi-path routing at a global level. So, BeeIP detect multiple paths from source to destination and distributes traffic across them in a scalable, robust and efficient way. Cross-layering is used in order to utilize parameters of lower layers and be able to calculate the performance of the links between the sources and the destinations.

These theories are designed to map the concepts of their natural, the sender node will act as being the hive, the destination node will act as being the source of food, and all the Intermediate nodes will consist of the path that a bee forager needs to traverse from one endpoint to the other.
BeeIP following four mechanisms, there are Adaptive Scouting, Adaptive Foraging, Optimal path selection, Detection of path failure.

\section{A) Adaptive Scouting:-}

While a scouting process is initialized in order to discover paths towards a given destination. When a route is required at the source node, a scout packet is making and sent by broadcast to the network. The main work is scout packet for determining available paths to its destination, while it introduces neighbouring nodes to each other, in a hop by hop manner. If the receiving node is desired destination an ack-scout packet is created and send back to the source node. Unicast is used instead of broadcast, because the ack-scout already knows which node to visit in order to return path. Additionally, the destination node assign to each discovered path a unique identification number, during journey back ack-scout constantly collect and deliver from one node to other node. A destination node is granted to create multiple ack-scouts depending on the number of scouts it receives from the network, allowing multiple paths to be established. On their way back, ack scouts recognize the success of the scouting to both the intermediate nodes and the source node.

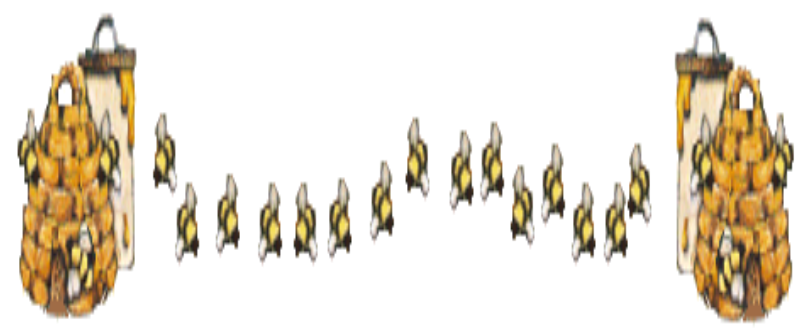

Figure.5. Scouting process

\section{B) Adaptive Foraging:-}

The scouting process is designed successful when one or more ack_scout is collected by the source node. When a forager is received by the destination it delivers continuous data packet and converts ack-forager. The ack_forager stays at the destination node before some data packet use to go back to the initial source. Then, proceed ack-forager are able to judge the quality of path and detect any improvement over time towards source. During travelling back home, it collects up-to-date information from the nodes it stay and the links between them. This process is used to monitor the overall path's quality and capable to report the finding back to the source. The path is seen as a group of links between the intermediate nodes, from the destination backward to the source. Quality of path is considered to signal strength, speed, energy, queue, delay etc, all the parameters to evaluate and monitoring the foraging process.

To calculate the quality of link from node, assume node $\mathrm{j}$, $\mathrm{k}$ and agent $\mathrm{b}$, w'-appropriate weight:

$$
\begin{gathered}
\mathrm{q}_{\mathrm{jk}}=\mathrm{sig}_{\mathrm{b}}^{\prime} * \mathrm{w}_{\mathrm{sig}}+\mathrm{speed}_{\mathrm{j}}^{\prime} * \mathrm{w}_{\text {speed }}+\text { energy }_{\mathrm{j}}^{\prime} * \mathrm{w}_{\text {energy }} \\
+\mathrm{qd}_{\mathrm{j}}^{\prime} * \mathrm{w}_{\mathrm{qk}}+\mathrm{txd}_{\mathrm{jk}}^{\prime} * \mathrm{w}_{\mathrm{txd}}
\end{gathered}
$$


Then to calculate the quality of path from destination $\mathrm{d}$ to the source s can be expressed formula on:

$$
\mathrm{Q}_{\mathrm{ds}}=\sum^{\mathrm{m}-1 \wedge \mathrm{n}=1}\left(\mathrm{q}_{\mathrm{Nn}+1 \rightarrow \mathrm{Nn}}\right), \quad\left[\mathrm{d}=\mathrm{N}_{\mathrm{m},}, \mathrm{s}=\mathrm{N}_{1}\right]
$$

Here $\mathrm{m}$ is the total number of nodes, $\mathrm{Nn}+1 \rightarrow \mathrm{Nn}$ is the pair of nodes with direction towards the source node.

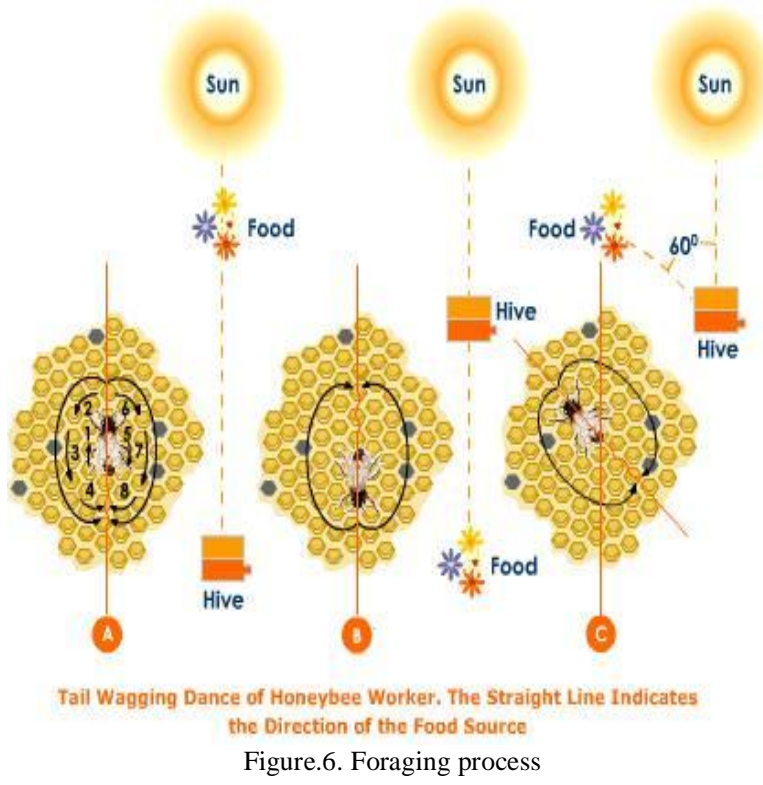

\section{C) Optimal Path Selection}

On their way back, foragers collect that information and record each path within a selection metric values and can be applied different selection metrics. Different Selection metrics are number of hops, energy, delay, speed, and signal strength etc. best path selection process rules on first calculate packet length and waiting for time in a queue until it can be executed from the destination towards source then it select fastest path from the list is selected.

This type of selection is done only at the source node and intermediate node preferred process is done using identification number and direction of path. At the destination node the selection is done using FIFO (Firstin-first out) fashion. Finally the select best path, data transmitted via intermediate node through destination.

\section{D) Path Failure}

BeeIP is designed 1

"link" level. When a link breakage within the path is detected no forager packet return back to the source node within a period of time then source node sets the path capacity to zero and marks the path as unacknowledged. The first establish that no future foragers will be given the broken path's ID and recent path to become available again, finally if a forager comes back. Furthermore, Timer used that purpose is unwanted information and old unacknowledged paths is removed. This simple mechanism ensure that control overhead also in terms of intermediate node and destination nodes, a timer pruning is also triggered and unwanted information is removed from path failure process.

\section{SIMULATION ANALYSIS}

Network Simulator (Version 2), widely known as NS2, is popular simulator in scientific environment. It is a discrete event simulator targeted at network research and focused on modeling network protocols such as ad hoc routing, sensor networks etc also NS2 is based on two languages. They are object oriented simulator $(\mathrm{C}++)$ and OTcl (object oriented Tcl) interpreter.

In this paper, we presented our research model testing result, which is shown in graph. Here we analyzed some performance metrics. Such as, Packet Delivery Ratio, Control overhead, Throughput, End-to-End Delay.

\begin{tabular}{|c|c|c|}
\hline S. No & Simulation parameters & values \\
\hline 1 & Channel type & $\begin{array}{c}\text { Channel / Wireless } \\
\text { channel }\end{array}$ \\
\hline 2 & $\begin{array}{c}\text { Radio Propagation } \\
\text { model }\end{array}$ & $\begin{array}{c}\text { Propagation / } \\
\text { TwoRayGround }\end{array}$ \\
\hline 3 & $\begin{array}{c}\text { Network interface Type } \\
\text { Phy } \\
\text { Wireless Phy }\end{array}$ \\
\hline 4 & Mac type & Mac 802_11 \\
\hline 5 & Antenna Model & $\begin{array}{c}\text { Antenna / Omni } \\
\text { antenna }\end{array}$ \\
\hline 6 & Max packet in ifq & 50 \\
\hline 7 & No of mobile nodes & $25-100$ \\
\hline 8 & Packet Size & 512 bytes \\
\hline 9 & Energy in joules & 10000 \\
\hline 10 & Routing protocol & $\begin{array}{c}\text { AODV AOMDV } \\
\text { AntHocNet, BeeIP }\end{array}$ \\
\hline
\end{tabular}

Table.1. Experimental set up in NS-2

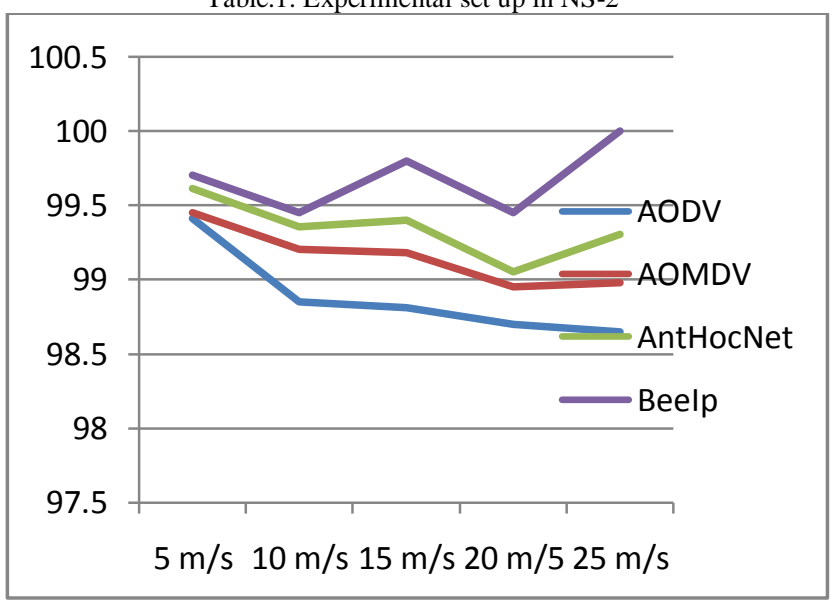

Figure.7.Different node Speeds Vs. Packet Delivry Ratio(\%)

\section{A) Packet Delivery Ratio}

Packet delivery ratio is defined as a number of packets successfully send and receive transmitted packets between source and Destination.

\section{B) Control Overhead}

This is the number of routing related packets that are send in order to exchange routing information and maintain routing. The less control overhead presented by proposed protocol. 


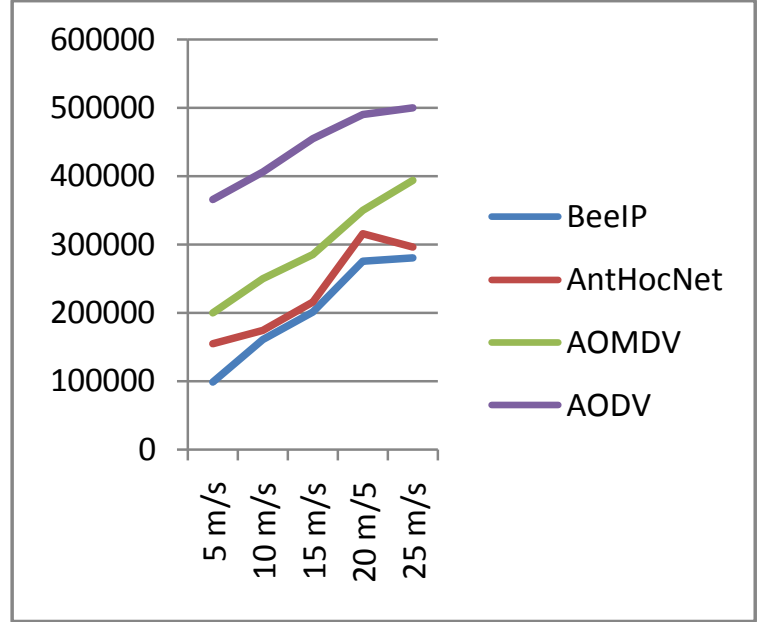

Figure.8 Different nodes Speeds vs. Number of Packets

\section{C) Throughput}

Throughput refers to the amount of data received by the destination node. However, how much data can be transferred from one location to another in a given amount of time.

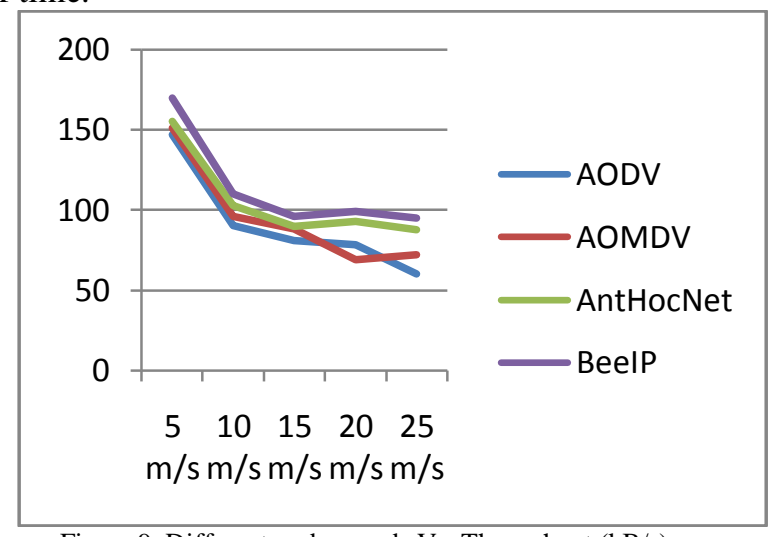

Figure.9. Different node speeds Vs. Throughput (kB/s)

D) End-to-End Delay

Average End-to-End delay is the average time of the data packet to be successfully transmitted from source to destination. It includes all possible delays such as propagation delay, queuing delays, process delays, etc. A small average End-to-End delay means faster data transmission .Average delay presented by proposed protocol.

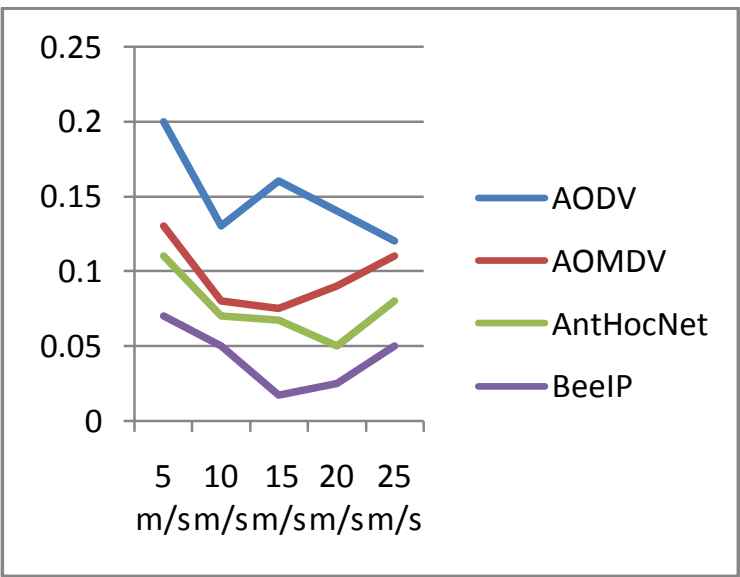

Figure.10 Different nodes speeds Vs. Delay (sec)

\section{CONCLUSION}

The proposed protocol BeeIP is able to faster data transmission and speed is very high and delay is very low compare than other SI protocol. Eventually, this protocol maintains balanced control overhead. Further, we analyze to enhance more secure data transmit process will achieve using algorithm.

\section{REFERENCES}

[1] C. Maddilet, Vennapusa Prakash Reddy, P. Jammulaiah "An Evaluation Study of Multipath Routing Protocols in AHWMNs" on International journal of Hardware and Software Research in Engineering , 2014

[2] MaahiAmitKhemchandani, Prof.B.W.Balkhande, "Comparative Analysis of AntHocNet, AODV, DSR Routing Protocols for Improvising Loss Packet Delivery Factor" on International Journal of Computer Science and Information Technologies (IJCSIT), Vol. 5 (5), 2014.

[3] Guido Falk von Rudorff, Christoph Wehmeyer, Daniel Sebastiani" Efficient implementation and application of the artificial bee colony algorithm to low-dimensional optimization problems", Computer Physics Communications 185 (2014) 1639-1646, 2013.

[4] Alexandors Giagkos et al "BeeIP-A swarm Intelligence based routing for wireless adhoc network" information science, Elsevier, 2013.

[5] M. Basu, "Artificial bee colony optimization for multi-area economic dispatch", Electrical Power and Energy Systems 49 (2013) 181-187, Elsevier, 2013.

[6] Muhammad Saleem, Israr Ullah, Muddassar Farooq "BeeSensor: An energy-efficient and scalable routing protocol for wireless sensor networks", Information Sciences 200 (2012) 38-56, ELESVIER, 2012. 